\title{
Effects of myofascial techniques on pain, mobility and function in patients with low back pain: a double-blind, controlled and randomized trial
}

\author{
Fabiana Forti Sakabe*1,3, Danielle Audickas Mazer², Júlia Alves $\mathrm{Cia}^{2}$, Daniel Iwai Sakabe ${ }^{1}$, Gustavo Luiz Bortolazzo ${ }^{3}$
}

1,2Faculdades Integradas Einstein de Limeira, Limeira (SP), Brazil, ${ }^{3}$ Colégio Brasileiro de Osteopatia, Sorocaba (SP), Brazil.

\begin{abstract}
Background: Low back pain is one of the most frequent causes of disability, with several associated etiologies. Osteopathic manipulative treatment is widely used to evaluate and manage musculoskeletal disorders. The aim of the study was to evaluate the immediate and late effects of 3 sessions of myofascial osteopathic techniques on pain intensity, posterior chain flexibility, lumbar mobility and level of disability in patients with chronic low back pain. Methods: 60 subjects of both genders, randomly divided into 3 experimental groups: healthy control (HC $n$ $=20$ ), low back pain control group $(\mathrm{LC}, \mathrm{n}=20)$ and treated low back pain group $(T G, n=20)$. Initially, the 3 groups were evaluated using the Visual Analogue Scale (VAS), Oswestry questionnaire, Wells bench and measurement of lateral spine tilt and fingertip-to-floor test. The TG was submitted to 3 sessions of myofascial techniques (lasting 40 minutes), 1x/week. The session consisted of the application of 6 myofascial techniques (thoracolumbar fascia, quadratus lumborum fascia, iliopsoas muscle stretching, quadratus lumborum stretching and iliolumbar ligaments pumping). Subjects were reevaluated immediately after the first session, 7 days after the last session and one month after treatment completion (follow up). Results: There was an improvement in posterior chain flexibility $(20.3 \pm 7.4 \mathrm{~cm}$ pre to $26.3 \pm 8 \mathrm{~cm}$ after 3 sessions), spinal mobility (fingertip-to-floor: $13.3 \pm 11.33 \mathrm{~cm}$ pre to $4.8 \pm 10.5 \mathrm{~cm}$ after 3 sessions), as well as pain intensity reduction $(3.3 \pm 1.9 \mathrm{~cm}$ pre to $1 \pm 1.7$ after 3 sessions) and reduction in the level of lumbar disability $(15.8 \pm 7.3$ in the pre to $9.2 \pm 8.6$ after 3 sessions) for TG. In HC and HC there was no change in any of the variables. The results shown for TG remained even one month after the intervention. Conclusion: The osteopathic treatment protocol with myofascial techniques was effective for the treatment of low back pain.

Keywords: Low back pain; Ostheopathic Medicine; Visual Analog Scale; Spine; Fascia.
\end{abstract}

\section{BACKGROUND}

According to Patrick et al. ${ }^{(1)}$ and Meucci et al. ${ }^{(2)}$ low back pain (LBP), also known as lower back pain, is characterized as severe or moderate pain between the 12th thoracic rib and the gluteal fold, with possible irradiation to the leg. Whether acute or chronic, it results from tissue damage, causing emotional and social damage. According to Gore et al. ${ }^{\left({ }^{(3)}\right.}$ and Schiphorst et al. ${ }^{(4)}$ chronic LBP affects about $11.9 \%$ of the world population, causing occupational incapacity leading to labor leave, generating financial losses for the state and health system, in addition to social and emotional losses (such as stress, anxiety and fear) ${ }^{(5)}$. It is a pain that persists for more than 3 months ( 12 weeks), with a multifactorial cause, such as pathological, sociodemographic, behavioral, ergonomic and emotional. LBP is considered a worldwide public health problem, affecting about 27 million adults in Brazil, according to IBGE (Brazilian Institute of Geography and Statistics) and it was the first cause of disability retirement in Brazil, in $2007^{(6)}$. Around 50 billion dollars a year are invested in LBP in expenses worldwide ${ }^{(7)}$. Some studies have pointed out as one of the risk factors for the appearance of LBP, the alteration of lumbar mobility and the decrease in flexibility, mainly of the muscles of the posterior chain. Flexibility is the physical capacity responsible for executing a movement of maximum angular amplitude of a joint or group of joints, respecting the physiological limits and avoiding the risk of injury. This physical capacity is mainly related to the postural habits, the elasticity of muscles and the plasticity of ligaments, tendons and joint capsules $^{(8)}$.
Physiotherapy in the treatment of LBP has the main objective of controlling pain, contributing to the well-being and quality of life (QOL) of the patient, and in this way, making it possible the return of the patient's functional activities. For this, Physiotherapy acts through conservative treatment and several procedures have been branching out, such as Manual Therapy, which arises the proposal for the application of Osteopathy ${ }^{(9)}$. In Osteopathic Medicine, the human body is seen as a functional unit, since all structures are connected by the fascial connective tissue, composed of collagen, elastin and hyaluronic acid fibers arranged in an irregular arrangement, allowing the functional performance of coating, containment and resistance to tension forces, and becoming essential for the transmission of muscle strength for synergistic motor coordination and for the proper positioning / functioning of the organs. The fascia is innervated by intrafascial mechanoreceptors, which, when stimulated, activate the autonomic system and cause changes in the tone and viscosity of the tissue, and transmit mechanical stimuli in painful information ${ }^{(10)}$. Osteopathy uses therapeutic manipulation techniques, based on the fact that the human body is able to readjust many pathologies through external help. It is based on body biomechanics, and may involve manipulations of joints, muscles, fascias, organs, or directed to body circulation, as well as seeking to restore nerve impulses ${ }^{(11)}$. Thus, osteopathic techniques can contribute to pain relief, improvement of mobility and QOL of the lumbar spine and, consequently, influencing the improvement of LBP. 


\section{OBJECTIVES}

To evaluate the immediate and late effect of 3 sessions of osteopathic techniques of myofascial release on the intensity of pain, lumbar mobility and degree of disability of subjects with LBP.

\section{METHODS}

The project was approved by the Ethics and Research Committee integraded faculties Einstein of Limeira with protocol number 2.677.349. The volunteers were informed about the experimental procedures to which they were submitted, as well as the fact that they did not affect their health, and all signed the Free and Informed Consent Form (ICF), according to the Resolution 466/12 of the National Health Council (CNS). The evaluation and intervention were carried out in the integraded faculties Einstein of Limeira. Sixty volunteers of both genders participated in the study, divided into 3 experimental groups: healthy control $(H C, n=20)$, LBP control group ( $L C, n=20$ ), treated LBP group ( $T G, n=20)$. $L C$ and TG had LBP for at least 3 months and were randomly divided by drawing. Inclusion criteria were age between 18 and 50 years and LBP for at least 3 months. Exclusion criteria were subjects with a diagnosis of disc extrusion, continuous use of analgesic medication, who were undergoing any other physiotherapeutic treatment and/or medication or general physical activities during the research period, who presented osteoporosis, history of recent musculoskeletal injuries, inability to understand the project, tumors and history of surgery in the LBP.

\section{Procedures}

Initially, the volunteers of the 3 groups were submitted to the evaluation of LBP intensity using the Visual Analog Scale (VAS). They received the scale printed on sulfite paper and were instructed to indicate, with the help of a pen, the intensity of the painful sensation at a point on the line, with scores ranging from 0 (zero) to 10 (ten). Then, the evaluator measured with a ruler, in millimeters, the distance between the left end, anchored by the words without pain and the point marked by the participant. After that, the volunteers answered the Oswestry Disability Index 2.0 Questionnaire. Each volunteer scored only one answer for each of the 10 questions.

In the sequence, the mobility of the spine (lateral inclination to the right and left) and the flexibility of the posterior chain (Wells and Dillon's Bench (sit and reach test) and fingertip-to-floor test) were measured. For the measurement of the fingertip-to-floor test, the volunteer barefoot on a floor without unevenness, was instructed to perform maximum flexion of the trunk, taking the fingers towards the floor, with the knees extended. Using a measuring tape, the examiner measured the distance from the third finger of the volunteer's left hand to the floor. To assess the lateral inclination of the spine, with the volunteer in the orthostatic position, they were asked to do the maximum lateral inclination to the right. At this point, the researcher measured the distance $(\mathrm{cm})$ from the third finger to the floor with the aid of a tape measure. Then, the same measurement was performed for the left side. To measure the flexibility of the posterior chain, it was used the Wells and Dillon's Bench. For this purpose, the volunteers remained seated on the floor, with lower limbs extended and feet parallel, resting on the surface of the bench, with torso leaning against a wall and looking towards the horizon. In this position, they were asked to tilt the torso and upper limbs forward, pushing the ruler of the equipment as far as possible, without flexing their knees. At the end of the test, the researcher noted on the form the value (in cm) achieved.

After the aforementioned assessments, volunteers in the LC underwent osteopathic intervention, which consisted of 3 sessions with an average duration of $\mathbf{4 0}$ minutes, $1 \mathrm{x}$ per week. For this, the volunteer used malleable clothing that did not restrict the range of motion. During the intervention, 6 osteopathic techniques (muscle, fascial or ligament) were performed as: stretching for the quadratus lumborum muscle, ligament pumping for the iliolumbar ligaments, lumbar myofascial release technique, fascia inhibition of the quadratus lumborum muscle, stretching for the iliopsoas muscle, stretching for the iliolumbar ligaments. Each technique was maintained for 90 seconds each side, except for the lumbar myofascial technique that was maintained for 120 seconds.

1) Technique of stretching for the quadratus lumborum muscle: the volunteer seated on the stretcher, the evaluator fixed the volunteer's pelvis against the stretcher, and positioned in trunk tilt and pulled the region of the last ribs upwards. This technique was performed bilaterally.

2) Technique of ligament pumping for the iliolumbar ligaments: supine position, therapist rested the volunteer's lower limb on the thigh and made contact, with one hand on the medial region of the thigh and, with the other hand on the posterior region of the iliac crest (close to the fixation of the iliolumbar ligaments). The technique consisted of pulling the patient's pelvis in the lower direction.

3) Lumbar myofascial release technique: prone position, therapist positioned the upper hand in the lumbosacral transitional vertebrae and the lower hand in the thoracolumbar transitional vertebrae. The technique consisted of moving the upper hand away from the lower hand, without letting the hands slide on the patient's skin.

4) Technique of fascia inhibition of the quadratus lumborum muscle: prone position, with the thigh on the side to be treated in adduction and the arm on the side to be treated in maximum abduction. The therapist pulled the lower limb in the lower direction and slid slowly and deeply over the lower back with the proximal hand. It was performed bilaterally.

5) Technique of stretching for the iliopsoas muscle: supine position on the stretcher, with one of the lower limbs out of the stretcher, the volunteer maintained the contralateral limb with triple flexion taking it towards the chest. The treated limb was taken in hip extension. In this position, the therapist performed slow and rhythmic movements towards the extension until the muscle tension 
was reduced. It was performed bilaterally.

6) Technique of lumbar inclination stretching (for the iliolumbar ligaments): lateral decubitus, with 90-degree flexion of the hips and knees. One hand made contact with the iliolumbar ligaments and the other hand held the volunteer's feet. The technique consisted of taking the volunteer's feet towards the ceiling, printing more lumbar inclination, in a slow and rhythmic way.

Immediately after performing the aforementioned maneuvers, each subject underwent a reassessment, using the VAS, and lateral inclinations, flexibility of the posterior chain and fingertip-to-floor testes, to determine if there was any immediate effect of the myofascial osteopathic techniques on LBP. Two more sessions were held, with an interval of 7 days between them. It is worth mentioning that in each session the volunteers went through the same tests before and after the interventions of each session, as previously mentioned. After 7 days and also 1 month after the end of the last session, the volunteers returned to the clinic for a final evaluation and reevaluation, respectively, in which the volunteers were assessed by spine mobility tests (fingertip-to-floor and lateral inclinations) and posterior chain flexibility. In addition, they answered the Oswestry and VAS questionnaire again. Two control groups were used as a means of data comparison: 20 volunteers in the group with LBP without treatment $(\mathrm{LC})$ and 20 healthy volunteers $(\mathrm{HC})$. All volunteers went through the evaluation protocol (except the HC, which answered the Oswestry questionnaire only in the first and last evaluations), with the same interval between sessions of the group that underwent the intervention.

For statistical analysis, Instat 3.0 software was used. Initially, the KS normality test was applied. As the data are normally distributed, the intragroup comparison was performed using the ANOVA test followed by Tukey's post-hoc test and for intergroup comparison, an unpaired test was used. In all calculations it was adopted the critical level of $5 \%$ $(p<0.05)$.

\section{RESULTS}

Table 1 shows the anthropometric characteristics of the studied volunteers. Regarding gender, 25 volunteers were male $(41.7 \%)$ and 35 were female $(58.3 \%)$. It can be seen in Table 1, that there was no significant difference between the groups evaluated, which represents sample homogeneity.

With regard to the flexibility of the posterior chain, measured with the aid of Wells and Dillon's Bench, it can be seen in Table 2 that in TG there was a significant improvement in flexibility after the intervention, in the different periods evaluated and that it remained in the follow up. In LC and HC there was no significant change in the periods evaluated. In the intergroup comparison, it can be seen that the $\mathrm{HC}$ presented significantly greater flexibility than the LC. In the periods after 3 sessions and follow up, the flexibility of the posterior chain of TG was similar to that of HC and significantly greater than that of LC. Regarding the spine mobility (in centimeters), the movements of fingertip-to-floor distance (table 2), right and left lateral inclination (table 3 ) were measured.
Table 2 shows the fingertip-to-floor distance of the studied volunteers. In TG there was a significant improvement in flexion mobility, since there was a significant reduction in the fingertip-to-floor distance in the different evaluated periods and the distance remained significantly smaller than in the pre-intervention period one month after the session. In $\mathrm{LC}$ and $\mathrm{HC}$, there was no significant change in the fingertip-tofloor distance in the different assessment periods. In the comparison of the 3 groups, it can be seen that after the 3 sessions and in the follow up period, the fingertip-to-floor distance of TG and HC was significantly shorter than LC, showing the treatment effectiveness in improving the spine mobility of TG. There was no significant difference between TG and $\mathrm{HC}$ after treatment and at follow up.

In the right and left lateral inclination (table 3), it was observed significant improvement in TG in the different evaluated periods in the follow up. Regarding the LC and HC, there was no significant change in the right and left inclination. In the comparison between the groups, there was a significant change only in the left lateral inclination between the TG and the LC, as the TG group showed a greater mobility of the left lateral inclination than the LC after the interventions.

With regard to the LBP intensity measured by the VAS, it can be seen in Table 4 that there was a significant reduction in TG in the different evaluated periods, which remained in the follow up. Regarding the LC, the data remained unchanged. In the comparison between TG and LC, the intensity of LBP in TG was significantly lower than in LC in the evaluated periods, demonstrating the effectiveness of osteopathic intervention in reducing the LBP intensity. Regarding the degree of disability promoted by LBP to perform activities of daily living, assessed by the Oswestry disability index, it can be seen that there was a significant improvement in this index in TG after the 3 osteopathic sessions and that it remained in the follow up (1 month after the end of the intervention). In the LC there was no significant change in the Oswestry questionnaire in the different periods evaluated, showing that the lumbar disability was maintained. When comparing the two groups, TG had a significantly lower questionnaire score than LC after 3 sessions and follow up, showing that the treatment was effective in reducing the degree of lumbar disability of the studied volunteers (table 4).

Table 1- Anthropometric variables

\begin{tabular}{|c|c|c|c|c|}
\hline & Age(years) & Weight(Kg) & Height(m) & BMI \\
\hline $\mathrm{HC}$ & $27,4 \pm 10,4$ & $67,8 \pm 15,3$ & $1,68 \pm 0,1$ & $\begin{array}{l}23,7 \pm \\
3,7\end{array}$ \\
\hline LC & $32,1 \pm 13,6$ & $69,9 \pm 16,1$ & $1,7 \pm 0,1$ & $\begin{array}{l}24,8 \pm \\
4,2\end{array}$ \\
\hline TG & $30,7 \pm 11,2$ & $76,2 \pm 21,1$ & $1,69 \pm 0,09$ & $\begin{array}{l}26,2 \pm \\
5,0\end{array}$ \\
\hline
\end{tabular}

Note: *Mean \pm standard deviation; body weight (Kg), height (meters), body mass index - BMI $\left(\mathrm{kg} / \mathrm{cm}^{2}\right)$; healthy control $(\mathrm{HC}, \mathrm{n}=20)$, low back pain control group ( $(L C, n=20)$, treated low back pain group (TG, $n=20), n=60$. 
Table 2 - Fingertip-to-floor and posterior chain flexibility tests

\begin{tabular}{lcccccc}
\hline & \multicolumn{4}{c}{ Fingertip-to-floor test (cm) } & \multicolumn{2}{c}{ Flexibility (cm) } \\
& HC & LC & TG & HC & LC & TG \\
\hline Pre & $8.3 \pm 12.5$ & $18 \pm 5$ & $13.3 \pm 11.33$ & $23.7 \pm 8.4^{€}$ & $16.2 \pm 4$ & $20.3 \pm 7.4$ \\
Post & & & $8.5 \pm 11.5^{*}$ & & & $24.3 \pm 7.6^{*}$ \\
Reevaluation & $6.5 \pm 11.2^{*} €$ & $17.9 \pm 4.8$ & $4.8 \pm 10.5^{* \#}$ & $22.3 \pm 9^{€}$ & $16.3 \pm 3.3$ & $26.3 \pm 8^{* \# €}$ \\
Follow up & $6 \pm 11^{€}$ & $18.2 \pm 5$ & $5.2 \pm 10.3^{* \# €}$ & $23.5 \pm 10.2^{€}$ & $17.2 \pm 4$ & $26.1 \pm 7.7^{* \# €}$ \\
\hline
\end{tabular}

Note: *Mean \pm standard deviation of fingertip-to-floor $(\mathrm{cm})$ and posterior chain flexibility $(\mathrm{cm})$ tests of healthy control (HC, $\mathrm{n}=20)$, low back pain control group ( $L C, n=20$ ), treated low back pain group ( $T G, n=20$ ) in different periods: pre (before treatment), post (immediately after the 1st session), reevaluation ( 7 days after the 3 rd session) and follow up (a month after treatment), $n=60 .{ }^{*} P<0.05$ regarding to pre of the respective group; $\# p<0.05$ regarding to post of TG; $€ p<0.05$ regarding to the respective period of LC.

Table 3 - Mobility of spine inclination test

\begin{tabular}{llcccc}
\hline & \multicolumn{2}{c}{ Right inclination (cm) } & \multicolumn{2}{c}{ Left inclination (cm) } \\
& HC & LC & TG & LC & TG \\
\hline Pre & $43.7 \pm 4$ & $43.3 \pm 2.9$ & $45 \pm 4$ & $44.9 \pm 2.9$ & $45.6 \pm 4.4$ \\
Post & & & $42 \pm 3.9^{*}$ & $42.8 \pm 3.4^{*}$ \\
Reevaluation & $41.4 \pm 5.3$ & $43.5 \pm 2.4$ & $40.9 \pm 4.7^{*}$ & $44.6 \pm 2.7$ & $40.9 \pm 4.2^{*}$ \\
Follow up & $42.7 \pm 4.2$ & $43.9 \pm 3$ & $41 \pm 2.5^{*}$ & $44.7 \pm 2.7$ & $41.7 \pm 3^{*}$ \\
\hline
\end{tabular}

Note: ${ }^{*}$ Mean \pm standard deviation of the mobility of spine inclination $(\mathrm{cm})$ of healthy control $(\mathrm{HC}, \mathrm{n}=20)$, low back pain control group (LC, $\left.\mathrm{n}=20\right)$, treated low back pain group (TG, $n=20$ ) in different periods: pre (before treatment), post (immediately after the 1st session), reevaluation ( 7 days after the 3 rd session) and follow up (a month after treatment), $n=60 .{ }^{*} \mathrm{P}<0.05$ regarding to pre of the respective group.

Table 4 - Low back pain intensity measured by visual analogue scale and the Oswestry Questionnaire.

\begin{tabular}{lcccc}
\hline & & Pain $(\mathrm{cm})$ & & \multicolumn{2}{c}{ Oswestry } \\
& LC & TG & LC & TG \\
\hline Pre & $4.5 \pm 1.3$ & $3.3 \pm 1.9$ & $16.2 \pm 4$ & $15.8 \pm 7.3$ \\
Post & & $1.11 \pm 1.4^{*}$ & $16.3 \pm 3.3$ & $9.2 \pm 8.6^{* \#}$ \\
Reevaluation & $4.4 \pm 1.1$ & $1 \pm 1.7^{* \#}$ & $17.2 \pm 4$ & $9 \pm 8.5^{* \#}$ \\
Follow up & $4.7 \pm 1$ & $0.9 \pm 0.9^{* \#}$ & & \\
\hline
\end{tabular}

Note: *Mean \pm standard deviation of low back pain intensity measured by visual analogue scale (cm), and the Questionnaire of healthy control (HC, $\mathrm{n}=20$ ), low back pain control group ( $L C, n=20$ ), treated low back pain group ( $T G, n=20$ ) in different periods: pre (before treatment), post (immediately after the 1st session), reevaluation ( 7 days after the 3 rd session) and follow up (a month after treatment), $n=60$. ${ }^{*} P<0.05$ regarding to the pre of the respective group; ${ }^{\mathrm{p}}<0.05$ regarding to the respective period of LC. 


\section{DISCUSSION}

According to the results obtained, it was found that the protocol was effective in reducing the intensity of LBP in the studied volunteers. As stated by Melzack and Wall ${ }^{(12)}$ the decrease in pain can be clarified by the gate control theory of pain, that is, cutaneous stimulation can cause pain relief through myofascial release techniques. The stimulus in the skin tends to neutralize the nociceptive afferents of small diameter nerve fibers when this stimulus is conducted by nerve fibers of a larger diameter, inhibiting pain at the level of the spine. According to Osborne ${ }^{(13)}$ several soft tissues are related to the appearance of LBP, especially the iliopsoas, quadratus lumbotum, paravertebral muscles and thoracolumbar fascia, causing, in addition to pain, a functional disability. As the proposed protocol included the release of these muscles, this factor may be responsible for reducing pain in the group that underwent intervention and consequently leading to a reduction in the degree of disability, as demonstrated by the Oswestry questionnaire. According to the same authors, nociceptive sensitization can be reduced through stimuli in the skin, such as the myofascial release techniques used in the study. The study of Martí-Salvador et al. ${ }^{(14)}$ demonstrated that osteopathic manipulative treatment protocols are effective in patients with LBP. Five sessions were held, twice a week lasting fortyfive minutes. It was used two groups, with isolated lumbar techniques and lumbar + diaphragm techniques. In view of the results, both groups had reduced pain (VAS and McGill) and lumbar disability (Oswestry and Roland Morris) after the intervention, and in the diaphragm group the gain was greater. These results corroborate those of the present study, since there was a reduction in the intensity of LBP and the degree of disability after 3 sessions of myofascial release in the group that underwent intervention, and the parameters remained reduced even a month after the end of the intervention.

Arguisuelas et al. ${ }^{(15)}$ applied an isolated myofascial release protocol in patients with chronic LBP. There were four sessions of myofascial treatment, lasting forty minutes. The results showed a significant improvement in relation to pain and the degree of disability caused by LBP. These results are in agreement with those of the present study, as well as the study of Licciardone et al. ${ }^{(16)}$ which demonstrated the effects of myofascial release techniques on chronic LBP, with a reduction in VAS and McGill.

In a study by Kariatsumari et al. ${ }^{(17)}$ it was found that a single session of manual therapy had positive effects on low back pain and mobility in athletes with LBP. There were 18 participants of both genders, aged between 15 and 17 years and complaining of LBP for at least four weeks in training and competitions. The treatment protocol consisted of applying myofascial release techniques and immediately afterwards they were reassessed by VAS and the modified Shober test. The study of Villalta Santos et al. ${ }^{(18)}$ evaluated 20 people with chronic LBP with visceral dysfunction, the protocol consisted of a 50-minute session of conventional physiotherapy and osteopathic visceral manipulations. Pain perception, lumbar mobility and functionality were assessed in three stages. 10: one week before the intervention, 2 : : immediately after the last intervention, 3: 1 week after the last intervention. Participants were divided into two groups, 10 for the experimental group (conventional physiotherapy and visceral manipulation) and 10 for the control group (conventional physiotherapy and visceral manipulation with placebo). As a result, it was obtained a significant reduction in the experimental group for lumbar mobility and specific functionality. The combination of visceral manipulation and conventional physical therapy showed significant differences between the groups for mobility of the lumbar spine and specific functionality. The improvement happened after 5 sessions once a week and it was maintained a week after the end of the treatment.

\section{CONCLUSION}

In view of the results obtained, it can be concluded that the proposed protocol, with 3 sessions of myofascial techniques, promoted the reduction of pain intensity, reduction of the degree of lumbar disability, improved flexibility of the posterior chain and the mobility of the spine of subjects with LBP, which lasted for a month after the end of treatment. When comparing the data collected after the intervention, the low back pain group showed a higher degree of lumbar disability and mobility and flexibility of the posterior chain, whose treatment should aim to reestablish these aspects.

AUTHOR'S CONTRIBUTION: FFS: advisor, writing of the article, application of assessment instruments, data analysis. DAM: undergraduate student, writing the article, applying the intervention. JAC: student of scientific initiation, writing of the article, application of the intervention. DIS: writing of the article, statistical analysis of the data. GLB: co-supervisor, writing the article.

FUNDING: Research Support and Scientific Initiation Program (PAPIC) of Faculdades Integradas Einstein de Limeira and Colégio Brasileiro de Osteopatia.

CONFLICT OF INTEREST: the authors declare no conflict of interest.

\section{REFERENCES}

1. Patrick N, Emanski E, Knaub MA. Acute and chronic low back pain. Med. Clin North Am. 2016;100(1):169-81.

2. Meucci RD, Fassa AG, Paniz VM, Silva MC, Wegman DH. Increase of chronic low back pain prevalence in a medium sized city of southern Brazil. BMC Musculoskelet Disord. 2013;14:155.

3. Gore M, Sadosky A, Stacey BR, Tai KS, Leslie D. The burden of chronic low back pain: clinical comorbidities, treatment patterns, and health care costs in usual care settings. Spine (Phila Pa 1976). 2012;37(11): E668-77.

4. Schiphorst Preuper HR, Reneman MF, Boonstra AM, et al. Relação entre fatores psicológicos e incapacidade de 
auto-relato com base no desempenho na dor lombar crônica. EurSpine J. 2008;17(11):1448-56.5.

5. Kamalikhah T, Morowatisharifabad MA, RezaeiMoghaddam F, Ghasemi M, Gholami-Fesharaki M, Goklani S. Alexander Technique Training Coupled Withan Integrative Model of Behavioral Prediction in Teachers with Low Back Pain. Iran Red Crescent Med J. 2016;18(9):e31218.

6. IBGE - Instituto Brasileiro de Geografia e Estatística. Pesquisa Nacional por Amostra de Domicílios. Síntese dos Indicadores de 2009. IBGE, 2010. 1-288 p.

7. Durante $\mathrm{H}$, Vasconcelos EC. Comparação do método Isostretching e cinesioterapia convencional no tratamento da lombalgia. SeminaCiencBiol Saúde. 2009;30(1):83-90.

8. Sacco ICN, Aliberti S, Queiroz BWC, et al. A influência da ocupação profissional na flexibilidade global na vida das trabalhadoras do setor têxtil de Blumenau - Santa Catarina. Saúde soc. Vol. 19 no. 2 São Paulo June 2010.

9. Lizier DT, Perez MVP, Sakata RK. Exercícios para o tratamento de lombalgia inespecífica. Ver. Bras. Anestesiol. 2012;62(6):838-46.

10. Vale JR, Carvalho HFB, Andrade VLA, Almeida LC. Effectiveness of the ostheopatic treatment in intestinal constipation: a systematic review. GED gastroenterol. endosc. 2017;36(2):68-76.

11. Machado VCV, Bittencourt DC. A conduta fisioterapêutica convencional e a osteopática no tratamento de pacientes com dor lombar crônica. Revista Contexto e Saúde. 2011;11(20):551-558.
12. Melzack R, Wall PD. Pain mechanisms: a new theory. Science. 1965;150(3699):971-979.

13. Osborne C. Ah, what relief! deep tissue sculpting for low back pain. Massage\&Bodywork. 2009;24(3):60.

14. Martí-Salvador $M$, Hidalgo-Moreno L, DoménechFernández J, Lisón JF, Arguisuelas MD. Osteopathic manipulative treatment including specific diaphragm techniques improves pain and disability in chronic nonspecific low back pain: a randomized trial. Archives of physical medicine and rehabilitation. 2018;99(9):17201729.

15. Arguisuelas M, Lisón J, Sánchez-Zuriaga D, MartínezHurtado I, Doménech-Fernández J. Effects of myofascial release in nonspecific chronic low back pain: a randomized clinical trial.Clinical biomechanics. 2019;63(1):27-33.

16. Licciardone JC, Kearns CM, Minotti DE. Outcomes of osteopathic manual treatment for chronic low back pain according to baseline pain severity: Results from the osteopathic trial. Revista Manual Therapy. 2013;18(6):533-40.

17. Fernandes GVB, Zeferino TV, Macedo CSG, Fernandes WVB. Efeito da terapia manual na dor e mobilidade lombar de atletas com lombalgia. Revista Ciência \& Saúde. 2009;7(31):181-185.

18. Santos LV, Córdoba LL, Lopes JBP, et al. Active visceral manipulation associated with convention al physiotherapy in people with chronic low back pain and visceral dysfunction: A preliminary, randomized, controlled, double-blindclinicaltrial. J Chiropr Med. 2019;18(2):79-89. 